\title{
Acquired factor VII deficiency associated with synovial sarcoma
}

\author{
Jarin Chindaprasirta ${ }^{\mathrm{a}}$, Chinadol Wanitpongpun ${ }^{\mathrm{b}}$, Piti Ungareewittaya ${ }^{\mathrm{c}}$ \\ ${ }^{a}$ Medical Oncology Division, ${ }^{b}$ Hematology Division, ${ }^{c}$ Pathological Department, Faculty of \\ Medicine, Khon Kaen University, Khon Kaen 40002, Thailand
}

\begin{abstract}
Background: Acquired factor VII (FVII) deficiency mostly results from liver disease or vitamin K antagonists. Isolated acquired FVII deficiency is a rare event, and only a few cases have been reported. To our knowledge, this is the first reported case of synovial sarcoma associated with FVII deficiency.

Objective: To describe our findings in a 20-year-old man, who presented with hemoptysis, weight loss, and later developed a suprascapular mass.

Methods: A review of the medical record of the patient plus a comparison with the literature was conducted.

Results: A biopsy from a suprascapular mass was made and the histopathological diagnosis of the tumor was confirmed as synovial sarcoma. Initial studies indicated a normal activated partial thromboplastin time and a prolonged prothrombin time. The FVII level was 31\%. No evidence of a FVII inhibitor or inactivator was demonstrated. Prothrombin time normalized during tumor regression and FVII level was elevated to $103 \%$.

Conclusion: Clinicians should be alert to the possible association of acquired FVII deficiency in cancer patients.
\end{abstract}

Keywords: Bleeding, factor VII deficiency, soft tissue sarcoma

Factor VII (FVII) is a vitamin K-dependent coagulation factor with a short circulating half-life. Acquired FVII deficiency mostly results from liver disease or vitamin $\mathrm{K}$ antagonists. Isolated acquired FVII deficiency is a rare event and only a few cases have been reported. The deficiency has been associated with aplastic anemia [1], severe sepsis $[2,3]$, and bone marrow transplantation [4]. A few cases were described in patients suffered from malignancies [5-10]. We present here a case in a 20-year-old man who was diagnosed as having acquired FVII deficiency and synovial sarcoma.

The patient had signed an informed consent form and ethics approval for this report was provided by Ethics Committee of the Medicine Faculty of Khon Kaen University following the guidelines of the Helsinki Declaration.

\section{Case report}

A 20-year-old man presented to a private hospital with complaints of nonmassive hemoptysis and weight loss. Chest X-ray imaging revealed abnormal findings

Correspondence to: Dr. Jarin Chindaprasirt, Division of Oncology, Internal Medicine Department, Faculty of Medicine, Khon Kaen University, Khon Kaen 40002, Thailand. E-mail: jarich@kku.ac.th and chest computed tomography (CT) was scheduled. Because of the flood crisis in Thailand in mid-2011, he did not undergo the CT. One month later, he complained of fullness in the abdomen and a left scapula mass was palpated. CT of the chest showed a complex mass in right hemithorax size $14.9 \times 14.4 \times$ $12.2 \mathrm{~cm}$ (Figure 1). CT scan of abdomen showed a mixed solid and cystic mass size $6.2 \times 4.3 \mathrm{~cm}$ at left suprarenal region with a partial compression of the left renal hilum (Figure 2). In view of these imaging studies, the patient was referred to a tertiary care center for further workup. There was an eightcentimeter soft tissue mass just above left scapula. It was firm, nontender, and fixed. Chest examination revealed decreased breath sound of the entire right lung and there was an ill-defined mass just above the umbilicus with negative bimanual palpation. Complete blood count and basic chemistry panels were normal.

Additional laboratory tests showed AFP $2.62 \mathrm{ng} /$ $\mathrm{ml}$, -hcG $0.37 \mathrm{ng} / \mathrm{ml}$, and LDH > 1,000 U/L. Hepatitis $\mathrm{B}$ and $\mathrm{C}$ profiles and anti-HIV antibodies were all negative. An incisional biopsy of the suprascapular mass was performed and CT guided biopsy was scheduled to obtain the tissue from right lung. A routine coagulogram was checked prior to this operation, and unexpectedly showed prolonged prothrombin time 
(PT) with normal activated partial thromboplastin time (aPTT). A mixing test was done that showed correctable levels at both 0 and 2 hours and FVII activity was decreased to $31 \%$ (normal $>50 \%$ ). Diagnosis of acquired FVII deficiency was made and the CT guided biopsy was cancelled. He has no personal or family history of coagulopathy or blood dyscrasia.

Microscopic examination of tissue from the suprascapular mass revealed a proliferation of small round cell tumors (Figure 3). Immunohistochemical staining was positive for CD99 (Figure 4) and Bcl-2, while staining for synaptophysin, LCA, CD20, $\kappa$ and $\lambda$ light-chain immunoglobulin, cytokeratin AE1/AE3, chromogranin and vimentin was negative. The proliferation fraction (Ki-67) was 50\%. The findings described above were compatible with a poorly differentiated synovial sarcoma. Subsequently, he received four cycles of combination chemotherapy of doxorubicin, cyclophosphamide, and vincristine. The back mass shrank after first cycle and was impalpable after four cycles. Prothrombin time normalized and factor VII level was elevated to $103 \%$ after three cycles of chemotherapy, with a total duration of three months. After tumor recurrence, there was no abnormality in the coagulogram. Since then, the patient has not experienced any hemoptysis.

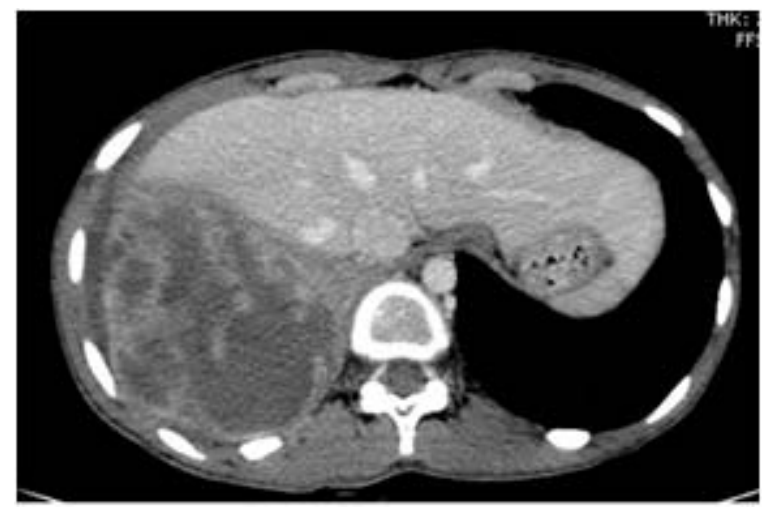

Figure 1. Chest CT scan revealed a heterodense mass located at right lower lung, size $14.9 \times 14.4 \times 12.2 \mathrm{~cm}$

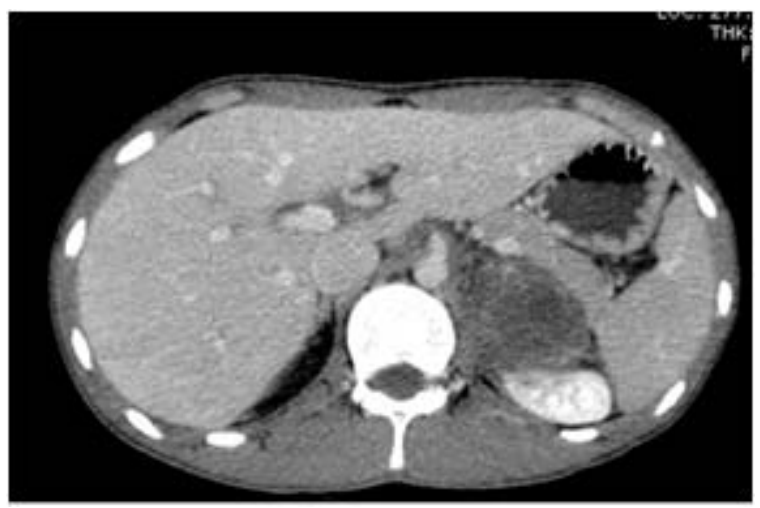

Figure 2. Abdominal CT scan revealed a left suprarenal mass 


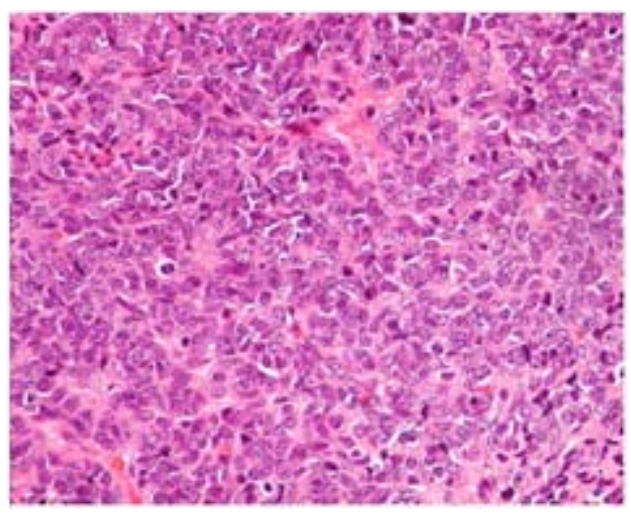

Figure 3. Tumor histopathology $(\times 400)$. The tumor consisted of numerous small round cell tumors (H\&E staining)

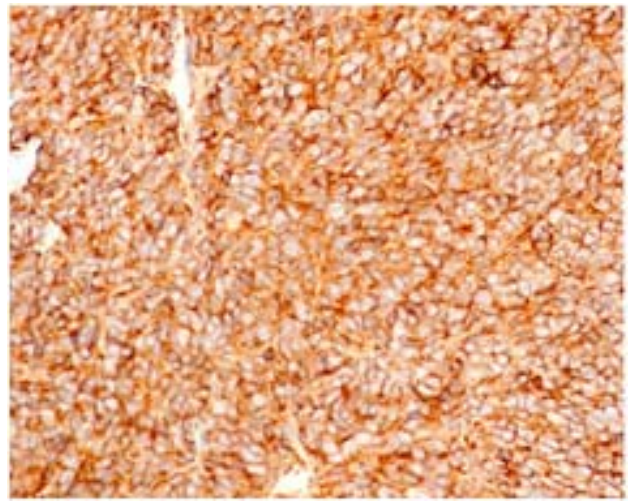

Figure 4. Tumor histopathology (×400). Membranes are diffusely immunopositive for CD99

\section{Discussion}

Acquired FVII deficiency in the absence of liver disease, vitamin K deficiency, drugs, and consumptive coagulopathy is a rare event. Only a small number of cases have been described in patients with solid organ malignancy and these are presented in Table $\mathbf{1}$.

The association of FVII level with hemorrhage is somehow not relevant. Our patient presented with hemoptysis, but FVII:C was 31\% while others in previous case reports were asymptomatic even though the FVII:C were much lower. By contrast, patients with sepsis and hematopoietic stem cells experienced more severe bleeding course and need more treatments to control the hemorrhage.

The mechanism of FVII deficiency in malignancy remains unclear. Mullighan et al. suggested two main reasons; FVII transient inhibitor or accelerated FVII clearance [11]. As in our case, the mixing test studies showed no verification of inhibitor. Nevertheless, some of the reported cases demonstrated inhibitor on the mixing studies.

Table 1. Case reports of isolated acquired factor VII deficiency in solid organ tumor

\begin{tabular}{lllllll}
\hline Author, year & Age, sex & Malignancy & FVII:C (\%) & Mixing study & Bleeding & Therapy \\
\hline Campbell et al., 1980 [5] & 66, male & Lung carcinoma & 30 & Inhibitor & None & No treatment \\
de Raucort et al., 1994 [6] & 30, male & Pleural liposarcoma & 15 & Inhibitor & None & IVIG, FFP \\
Hirshberg et al., 1994 [7] & 57, female & Uterine carcinoma & NS & NS & None & No treatment \\
Aguilar et al., 2003 [8] & 80, male & TCC bladder & 38 & Inhibitor & Gut, urinary & Corticosteroids \\
Zili et al., 2005 [9] & 63, male & Lung carcinoma & 6 & Deficiency & None & No treatment \\
Granger et al, 2009 [10] & 2, female & Wilm’s tumor & 17 & Deficiency & None & FFP
\end{tabular}

NS = not stated, TCC = transitional cell carcinoma, FVII:C = factor VII coagulant activity, IVIG = intravenous immunoglobulin, $\mathrm{FFP}=$ fresh frozen plasma 
Weisdorf et al. reported a case with aplastic anemia and FVII deficiency, which was corrected with primary disease definite treatment-bone marrow transplantation [1]. In our patient, his FVII level recovered upon tumor response to chemotherapy without any other treatment. This suggested that FVII deficiency is related to malignancy, although further investigation is needed to explore the mechanism.

The authors have no conflict of interest to declare.

\section{References}

1. Weisdorf D, Hasegawa D, Fair DS. Acquired factor VII deficiency associated with aplastic anaemia: correction with bone marrow transplantation. $\mathrm{Br} \mathrm{J}$ Haematol. 1989; 71:409-13.

2. Bidet A, Boiteux-Vergnes C, Mouton C. Acquired and repeated factor VII deficiency during two infectious episodes: a case report. Ann Biol Clin. 2009; 67:587-9.

3. Biron C, Bengler C, Gris JC, Schved JF. Acquired isolated factor VII deficiency during sepsis. Haemostasis. 1997; 27:51-6.

4. Acquired factor VII deficiency in hematopoietic stem cell transplant recipients. Bone Marrow Transplant. 2002; 29:403-8.
5. Campbell E, Sanal S, Mattson J, Walker L, Estry S, Mueller L, et al. Factor VII inhibitor. Am J Med. 1980; 68:962-4.

6. de Raucourt E, Dumont MD, Tourani JM, Hubsch JP, Riquet M, Fischer AM. Acquired factor VII deficiency associated with pleural liposarcoma. Blood Coagul Fibrinolysis. 1994; 5:833-6.

7. Hirshberg AJ, Fletcher RL, Harris Jr C, Dupper RL. Case report of factor VII deficiency. Am J Emerg Med. 1993;11:600-1.

8. Zili M, Hammami S, Marghli A, Mechmech L, Boughnim L, Abid S, et al. Acquired isolated factor VII deficiency and bronchogenic carcinoma. A case report. Tunis Med. 2005; 83:363-5.

9. Aguilar C, Lucia JF, Hernandez P. A case of an inhibitor autoantibody to coagulation factor VII. Haemophilia. 2003; 9:119-20.

10. Granger J, Gidvani VK. Acquired factor VII deficiency associated with Wilms tumor. Pediatr Blood Cancer. 2009; 52:394-5.

11. Mullighan CG, Rischbieth A, Duncan EM, Lloyd JV. Acquired isolated factor VII deficiency associated with severe bleeding and successful treatment with recombinant factor VIIa (NovoSeven). Blood Coagul Fibrinolysis. 2004; 15:347-51. 